\title{
Equilibrium States and Their Macroscopic Uniformity in Fermion Lattice Systems
}

\author{
Nobuyuki AKIHO \\ Graduate School of Information Sciences, Tohoku University, Aoba-ku, Sendai 980-8579, Japan \\ E-mail:akiho@ims.is.tohoku.ac.jp
}

\begin{abstract}
We study equilibrium states and their macroscopic uniformity in the fermion lattice system satisfying the assumptions presented by Araki and Moriya [5]. We show that every translation-invariant KMS state of the even part of the fermion algebra extends uniquely to a translation-invariant KMS state of the whole fermion algebra. The macroscopic uniformity is established for an extremal translation-invariant equilibrium state of the fermion algebra.
\end{abstract}

KEYWORDS: fermion algebra, potentials, KMS states, Gibbs condition, macroscopic uniformity

\section{Introduction}

The fermion lattice system is obtained as the $\mathrm{C}^{*}$-algebra generated by annihilation and creation operators on lattice sites of $\mathbb{Z}^{v}, v$ being a positive integer. We assume throughout this paper that $\mathcal{A}$ is the fermion algebra and $\alpha$ is a given strongly continuous one-parameter automorphism group of $\mathcal{A}$ satisfying the following assumptions presented in [5]:

(I) $\alpha_{t} \Theta=\Theta \alpha_{t}$ for all $t \in \mathbb{R}$, where $\Theta$ is the parity automorphism of $\mathcal{A}$.

(II) $\mathscr{A}_{0} \subset \mathscr{D}\left(\delta_{\alpha}\right)$, where $\mathcal{A}_{0}$ is the union of all local algebras and $\delta_{\alpha}$ is the generator of $\alpha_{t}$ with domain $\mathscr{D}\left(\delta_{\alpha}\right)$.

(III) $\mathcal{A}_{0}$ is a core for $\delta_{\alpha}$.

(IV) $\alpha_{t} \tau_{k}=\tau_{k} \alpha_{t}$ for all $t \in \mathbb{R}$ and $k \in \mathbb{Z}^{v}$, where $\tau$ is the lattice translation automorphism group of $\mathcal{A}$.

In Section 3, using von Neumann's measurable cross-section principle and the chemical potential theory [4], we show

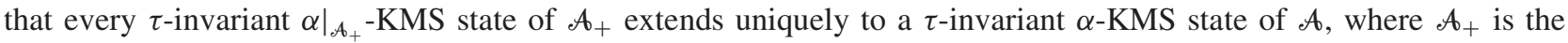
even part of $\mathcal{A}$. There are several corollaries of the above result. First, the extreme boundary of the simplex of $\tau$ invariant $\alpha$-KMS states of $\mathcal{A}$ is contained in that of the simplex of $\tau$-invariant states of $\mathcal{A}$. Next, restricting to the case $v=1$, we assume that $\Phi$ is a $\tau$-invariant potential in $\mathcal{A}_{+}$satisfying the following conditions:

$$
\sum_{\mathrm{K} \ni 0}\|\Phi(\mathrm{K})\|<\infty \quad \text { and } \quad \sup _{n \in \mathbb{N}}\left\|W_{\Phi}([1, n])\right\|<\infty
$$

where $W_{\Phi}([1, n])$ denotes the surface energy for the interval $[1, n]:=\{1, \ldots, n\}$ of $\mathbb{Z}$. Under the above conditions, $\Phi$ generates the one-parameter dynamics $\alpha^{\Phi}$ on $\mathcal{A}$ and it is well-known [2,12] that $\left(\mathcal{A}, \alpha^{\Phi}\right)$ has no phase transition. We see that $\mathcal{A}_{+}$has a unique $\left.\alpha^{\Phi}\right|_{\mathcal{A}_{+}}$-KMS state. This seems meaningful because the local algebras are always full matrix algebras in the existing literatures showing no phase transition while local algebras of $\mathcal{A}_{+}$are not.

Hiai and Petz studied the macroscopic uniformity for extremal equilibrium states in a spin lattice system [10]. Macroscopic uniformity is a basic feature of statistical mechanical systems (see [10, Sect. 3] for details). In Section 4 we establish the macroscopic uniformity for such states in the fermion lattice system as well.

Appendix is a simple remark concerning the correspondence between $\tau$-invariant potentials having short range and their standard variants introduced in [5].

\section{Preliminaries}

The definitions and results in this section are entirely based on [5].

\subsection{Fermion algebras}

By the fermion algebra $\mathcal{A}$, we mean a unital $\mathrm{C}^{*}$-algebra generated by $a_{i}$ and $a_{i}^{*}, i \in \mathbb{Z}^{v}$, satisfying the canonical anticommutation relations:

$$
\left\{a_{i}^{*}, a_{j}\right\}=\delta_{i j} \mathbf{1}, \quad\left\{a_{i}^{*}, a_{j}^{*}\right\}=\left\{a_{i}, a_{j}\right\}=0, \quad i, j \in \mathbb{Z}^{v},
$$

where $a_{i}^{*}$ is the adjoint of $a_{i},\{x, y\}:=x y+y x, \delta_{i j}:=1$ if $i=j$ and $\delta_{i j}:=0$ otherwise. The elements $a_{i}$ and $a_{i}^{*}$ are called annihilation and creation operators, respectively. Let $\mathcal{A}_{0}$ be the ${ }^{*}$-algebra generated by all $a_{i}$ and $a_{i}^{*}, i \in \mathbb{Z}^{v}$. For each $\mathrm{I} \subset \mathbb{Z}^{v}$, the $\mathrm{C}^{*}$-subalgebra of $\mathcal{A}$ generated by $a_{i}$ and $a_{i}^{*}, i \in \mathrm{I}$, is denoted by $\mathcal{A}(\mathrm{I})$. If the cardinality $|\mathrm{I}|$ of $\mathrm{I}$ is finite, then $\mathcal{A}(\mathrm{I})$ is referred to as the local algebra for $\mathrm{I}$. We set $\mathcal{A}(\emptyset):=\mathbb{C} \mathbf{1}$. The parity automorphism $\Theta$ of $\mathcal{A}$ is defined by $\Theta\left(a_{i}\right):=-a_{i}$ for all $i \in \mathbb{Z}^{v}$. The even and odd parts of $\mathcal{A}$ are defined as 


$$
\mathcal{A}_{+}:=\{a \in \mathcal{A}: \Theta(a)=a\}, \quad \mathcal{A}_{-}:=\{a \in \mathcal{A}: \Theta(a)=-a\},
$$

respectively.

The translation $\tau_{k}$ by $k \in \mathbb{Z}^{v}$ is an automorphism of $\mathcal{A}$ defined by $\tau_{k}\left(a_{i}\right):=a_{i+k}$ for all $i \in \mathbb{Z}^{v}$. A state $\omega$ of $\mathcal{A}$ is said to be even if $\omega \circ \Theta=\omega$. We denote by $\mathfrak{S}(\mathcal{A})$ the state space of $\mathcal{A}$ and by $\mathfrak{S}_{\tau}(\mathcal{A})$ the set of all $\tau$-invariant states of $\mathcal{A}$. If $X$ is a convex set in $\mathfrak{S}(\mathcal{A})$, we call the set of extreme points of $X$ the extreme boundary of $X$ and denote it by $\mathbb{E} X$.

The even part $\left(\mathcal{A}_{+},\left.\tau\right|_{\mathcal{A}_{+}}\right)$is asymptotically abelian in the norm sense while $(\mathcal{A}, \tau)$ is not. In fact, we note that $a \in \mathcal{A}_{+}$ if and only if the following asymptotically central property holds:

$$
\lim _{|k| \rightarrow \infty}\left\|\left[\tau_{k}(a), b\right]\right\|=0 \quad \text { for all } b \in \mathcal{A},
$$

where $[x, y]:=x y-y x$. If $\omega \in \mathfrak{S}_{\tau}(\mathcal{A})$, then $\omega$ is even $[6,5.2 .21]$ (see also [13]).

\subsection{Potentials and derivations}

For any $\mathrm{I} \subset \mathbb{Z}^{v}$, there exists a unique conditional expectation $E_{\mathrm{I}}$ from $\mathcal{A}$ onto $\mathcal{A}(\mathrm{I})$ such $\operatorname{that} \operatorname{tr}(a b)=\operatorname{tr}\left(E_{\mathrm{I}}(a) b\right)$ for all $a \in \mathcal{A}$ and $b \in \mathcal{A}(\mathrm{I})$, where tr denotes the unique tracial state of $\mathcal{A}$. We denote by $\mathscr{F}$ the family of all finite subsets of $\mathbb{Z}^{v}$. A mapping $\Phi$ from $\mathscr{F}$ into $\mathcal{A}$ is called a standard potential if it satisfies the following conditions:

(P1) $\Phi(\mathrm{I}) \in \mathcal{A}(\mathrm{I}), \Phi(\emptyset)=0$.

(P2) $\Phi(\mathrm{I})^{*}=\Phi(\mathrm{I})$

(P3) $\Theta(\Phi(\mathrm{I}))=\Phi(\mathrm{I})$.

(P4) $E_{\mathrm{J}}(\Phi(\mathrm{I}))=0$ if $\mathrm{J} \subset \mathrm{I}$ and $\mathrm{J} \neq \mathrm{I}$.

(P5) For each $\mathrm{I} \in \mathscr{F}$, the net $H_{\Phi}^{\mathrm{J}}(\mathrm{I}):=\sum_{\mathrm{K}}\{\Phi(\mathrm{K}): \mathrm{K} \cap \mathrm{I} \neq \emptyset, \mathrm{K} \subset \mathrm{J}\}$ is a Cauchy net in the norm topology of $\mathcal{A}$ for $\mathrm{J} \nearrow \mathbb{Z}^{v}$.

The real vector space of all standard potentials is denoted by $\mathcal{P}$. We denote by $\mathcal{P}_{\tau}$ the set of all $\Phi \in \mathcal{P}$ satisfying the following condition:

(P6) $\tau_{k}(\Phi(\mathrm{I}))=\Phi(\mathrm{I}+k)$ for all $\mathrm{I} \in \mathscr{F}$ and $k \in \mathbb{Z}^{v}$.

For each $\Phi \in \mathcal{P}$ and $\mathrm{I} \in \mathscr{F}$, define the standard Hamiltonian $H_{\Phi}(\mathrm{I})$ by

$$
H_{\Phi}(\mathrm{I}):=\lim _{\mathrm{J} \nearrow \mathbb{Z}^{\nu}} H_{\Phi}^{\mathrm{J}}(\mathrm{I})=\sum_{\mathrm{K} \cap \mathrm{I} \neq \emptyset} \Phi(\mathrm{K}),
$$

the internal energy $U_{\Phi}(\mathrm{I})$ by

$$
U_{\Phi}(\mathrm{I}):=\sum_{\mathrm{K} \subset \mathrm{I}} \Phi(\mathrm{K})
$$

and the surface energy $W_{\Phi}(\mathrm{I})$ by

$$
W_{\Phi}(\mathrm{I}):=\sum_{\mathrm{K}}\left\{\Phi(\mathrm{K}): \mathrm{K} \cap \mathrm{I} \neq \emptyset, \mathrm{K} \cap \mathrm{I}^{c} \neq \emptyset\right\}
$$

It follows that $\mathcal{P}_{\tau}$ is a separable real Banach space with the norm $\|\Phi\|:=\left\|H_{\Phi}(\{0\})\right\|$. If $\Phi \in \mathcal{P}_{\tau}$, then

$$
\left\|U_{\Phi}(\mathrm{I})\right\| \leq\left\|H_{\Phi}(\mathrm{I})\right\| \leq\|\Phi\| \cdot|\mathrm{I}| \cdot
$$

For each $\mathrm{I} \in \mathscr{F}$, we call the largest distance between two points in I the diameter of I. A standard potential $\Phi$ has finite range if $\Phi(\mathrm{I})=0$ for all $\mathrm{I}$ of sufficiently large diameter. We denote by $\mathcal{P}_{\tau}^{\mathrm{f}}$ the subspace of all $\Phi \in \mathcal{P}_{\tau}$ having finite range. Then $\mathcal{P}_{\tau}^{\mathrm{f}}$ is dense in $\mathcal{P}_{\tau}$.

The real vector space of all ${ }^{*}$-derivations with domain $\mathcal{A}_{0}$ and commuting with $\Theta$ on $\mathcal{A}_{0}$ is denoted by $\Delta\left(\mathcal{A}_{0}\right)$. We denote by $\Delta_{\tau}\left(\mathcal{A}_{0}\right)$ the real vector space of all $\delta \in \Delta\left(\mathcal{A}_{0}\right)$ commuting with $\tau$. For every $\Phi \in \mathcal{P}$, define a *-derivation $\delta_{\Phi}$ with domain $\mathcal{A}_{0}$ by

$$
\delta_{\Phi}(a):=i\left[H_{\Phi}(\mathrm{I}), a\right], \quad a \in \mathcal{A}(\mathrm{I}) .
$$

The equations (2.2) and (2.4) between $\Phi \in \mathcal{P}_{\tau}$ and $\delta_{\Phi} \in \Delta_{\tau}\left(\mathcal{A}_{0}\right)$ gives a one-to-one real linear mapping from $\mathcal{P}_{\tau}$ onto $\Delta_{\tau}\left(\mathscr{A}_{0}\right)$.

For a dynamics $\alpha$ given in Introduction, the *-derivation $\delta \in \Delta_{\tau}\left(\mathscr{A}_{0}\right)$ is determined uniquely by $\delta:=\left.\delta_{\alpha}\right|_{\mathcal{A}_{0}}, \delta_{\alpha}$ being the generator of $\alpha_{t}$. Moreover, for the ${ }^{*}$-derivation $\delta$, the $\tau$-invariant standard potential $\Phi$ is determined uniquely by the one-to-one correspondence mentioned in the preceding paragraph.

\subsection{Variational principle}

Let $\mathrm{I} \in \mathscr{F}$ and $\omega \in \mathfrak{S}(\mathcal{A})$. We shall denote the restriction of $\omega$ to $\mathcal{A}(\mathrm{I})$ by $\omega_{\mathrm{I}}$. The von Neumann entropy $S\left(\omega_{\mathrm{I}}\right)$ of $\omega_{\mathrm{I}}$ is defined by 


$$
S\left(\omega_{\mathrm{I}}\right):=-\omega\left(\log \frac{\mathrm{d} \omega_{\mathrm{I}}}{\mathrm{d} \operatorname{Tr}_{\mathrm{I}}}\right),
$$

where $\operatorname{Tr}_{I}$ denotes the canonical trace on $\mathcal{A}(\mathrm{I})$ such that $\operatorname{Tr}_{\mathrm{I}}(e)=1$ for all minimal projections $e$ in $\mathcal{A}(\mathrm{I})$. If $\omega \in \mathfrak{S}_{\tau}(\mathcal{A})$ and $\Phi \in \mathcal{P}_{\tau}$, then the following van Hove limits exist:

$$
\begin{aligned}
s(\omega) & :=\text { v.H. } \lim _{\mathrm{I} \rightarrow \mathbb{Z}^{v}} \frac{1}{|\mathrm{I}|} S\left(\omega_{\mathrm{I}}\right), \\
P(\Phi) & :=\text { v.H. } \lim _{\mathrm{I} \rightarrow \mathbb{Z}^{v}} \frac{1}{|\mathrm{I}|} \log \operatorname{Tr}_{\mathrm{I}}\left(e^{-H_{\Phi}(\mathrm{I})}\right)=\text { v.H. } \lim _{\mathrm{I} \rightarrow \mathbb{Z}^{v}} \frac{1}{|\mathrm{I}|} \log \operatorname{Tr}_{\mathrm{I}}\left(e^{-U_{\Phi}(\mathrm{I})}\right), \\
e_{\Phi}(\omega) & :=\text { v.H. } \lim _{\mathrm{I} \rightarrow \mathbb{Z}^{v}} \frac{1}{|\mathrm{I}|} \omega\left(H_{\Phi}(\mathrm{I})\right)=\text { v.H. } \lim _{\mathrm{I} \rightarrow \mathbb{Z}^{v}} \frac{1}{|\mathrm{I}|} \omega\left(U_{\Phi}(\mathrm{I})\right) .
\end{aligned}
$$

We refer to $s(\omega), P(\Phi)$ and $e_{\Phi}(\omega)$ as the mean entropy, pressure and mean energy, respectively. Define

$$
\Lambda(\Phi):=\left\{\omega \in \mathfrak{S}_{\tau}(\mathcal{A}): P(\Phi)=s(\omega)-e_{\Phi}(\omega)\right\} .
$$

It follows that $\Lambda(\Phi)$ is non-empty. When $\omega \in \Lambda(\Phi)$, we say that $\omega$ satisfies the variational principle with respect to $\Phi$.

\section{Translation-Invariant KMS States}

For simplicity we consider only $(\alpha, \beta)$-KMS states with $\beta=1$ and refer to those states as $\alpha$-KMS states; obviously all our results hold for any $\beta$ without change. Let $K_{\tau}(\mathcal{A})$ be the set of all $\tau$-invariant $\alpha$-KMS states of $\mathcal{A}$. By (IV) and (2.1), $\mathcal{A}_{+}$is invariant under $\alpha$, so that we can consider an $\left.\alpha\right|_{\mathcal{A}_{+}}$-KMS state of $\mathcal{A}_{+}$. We denote by $K_{\tau}\left(\mathcal{A}_{+}\right)$the set of all $\tau$ invariant $\left.\alpha\right|_{\mathcal{A}_{+}}$-KMS states of $\mathcal{A}_{+}$and by $\mathfrak{S}_{\tau}\left(\mathcal{A}_{+}\right)$the simplex of all $\tau$-invariant states of $\mathcal{A}_{+}$. For each $\omega \in K_{\tau}\left(\mathcal{A}_{+}\right)$, let $\left(\pi_{\omega}, \mathscr{H}_{\omega}, \Omega_{\omega}\right)$ be the cyclic representation of $\mathcal{A}_{+}$induced by $\omega$ and $u_{\omega}$ a unitary representation of $\mathbb{Z}^{v}$ implementing $\tau$ on $\mathscr{H}_{\omega}$. Since $\mathcal{A}_{+}$is asymptotically abelian with respect to $\tau$ due to $(2.1)$, it follows from $[6,4.3 .14]$ that

$$
\pi_{\omega}\left(\mathcal{A}_{+}\right)^{\prime} \cap u_{\omega}\left(\mathbb{Z}^{\nu}\right)^{\prime} \subset \pi_{\omega}\left(\mathcal{A}_{+}\right)^{\prime \prime} \cap \pi_{\omega}\left(\mathcal{A}_{+}\right)^{\prime} .
$$

Hence $K_{\tau}\left(\mathcal{A}_{+}\right)$is a face of $\mathfrak{S}_{\tau}\left(\mathcal{A}_{+}\right)$, and so it is a simplex. Moreover, it is obvious from (3.1) and [17, Lemma 4.7] that

$$
\varepsilon K_{\tau}\left(\mathcal{A}_{+}\right) \subset \mathcal{E S _ { \tau }}\left(\mathcal{A}_{+}\right) \text {. }
$$

For simplicity we shall denote the restriction of a state $\rho$ of $\mathcal{A}_{\text {to }} \mathcal{A}_{+}$by $\rho_{+}$.

Theorem 3.1. If $\omega \in K_{\tau}\left(\mathcal{A}_{+}\right)$, then there exists a unique $\bar{\omega} \in K_{\tau}(\mathcal{A})$ such that $\bar{\omega}_{+}=\omega$.

Proof. The proof is divided into four steps.

Step 1. We consider the extremal decomposition of $\omega$. Since $K_{\tau}\left(\mathcal{A}_{+}\right)$is a simplex, it follows from the Choquet-Meyer theorem [15] that there exists a unique probability measure $\mu$ concentrated on $E K_{\tau}\left(\mathscr{A}_{+}\right)$such that

$$
\omega(a)=\int_{\varepsilon K_{\tau}\left(\mathscr{A}_{+}\right)} \psi(a) \mathrm{d} \mu(\psi), \quad a \in \mathcal{A}_{+} .
$$

Step 2. We show that every $\psi \in \mathscr{E} K_{\tau}\left(\mathcal{A}_{+}\right)$extends to a weakly $\tau$-clustering state of $\mathcal{A}$. For each $\psi \in \mathcal{E} K_{\tau}\left(\mathcal{A}_{+}\right)$, define

$$
X_{\psi}:=\left\{\rho \in \mathfrak{S}_{\tau}(\mathcal{A}): \rho_{+}=\psi\right\} .
$$

We prove that $X_{\psi}$ is non-empty. First, there is a state $\bar{\psi}$ of $\mathcal{A}$ such that $\bar{\psi}_{+}=\psi$. Let $M$ be a Banach limit for bounded sequences. Since $\left\{\bar{\psi}\left(\tau^{n}(a)\right)\right\}$ is a bounded sequence for all $a \in \mathcal{A}$, we can define the $\tau$-invariant state $\tilde{\psi}$ of $\mathcal{A}$ by

$$
\tilde{\psi}(a):=M\left(\left\{\bar{\psi}\left(\tau^{n}(a)\right)\right\}\right), \quad a \in \mathcal{A} .
$$

If $a \in \mathcal{A}_{+}$, then $\tilde{\psi}(a)=\psi(a)$ due to $\tau^{n}(a) \in \mathcal{A}_{+}$.

Since $X_{\psi}$ is a non-empty weak ${ }^{*}$ compact convex set in $\mathfrak{S}(\mathcal{A}), X_{\psi}$ has an extreme point $\phi$ due to the Krein-Milman theorem. Let $\phi=\lambda \phi_{1}+(1-\lambda) \phi_{2}$ with $\phi_{1}, \phi_{2} \in \mathfrak{S}_{\tau}(\mathcal{A})$ and $0<\lambda<1$. Since $\phi_{1+}, \phi_{2+} \in \mathfrak{S}_{\tau}\left(\mathcal{A}_{+}\right)$while $\psi \in$ $\varepsilon \mathfrak{S}_{\tau}\left(\mathcal{A}_{+}\right)$due to (3.2) and $\psi=\phi_{+}=\lambda \phi_{1+}+(1-\lambda) \phi_{2+}$, we obtain $\phi_{1+}=\phi_{2_{+}}=\psi$ and so $\phi_{1}, \phi_{2} \in X_{\psi}$. Since $\phi \in \mathcal{E} X_{\psi}$, we obtain $\phi_{1}=\phi_{2}=\phi$; hence $\phi \in \mathcal{E S}_{\tau}(\mathcal{A})$. Since an extremal $\tau$-invariant state of the fermion algebra is weakly $\tau$-clustering due to [4, Sect. II.8], $\phi$ is a weakly $\tau$-clustering state of $\mathcal{A}$.

Step 3. We show that $\phi$ constructed in Step 2 is an $\alpha$-KMS state of $\mathcal{A}$. Let $\mathbb{Z}_{2}:=\mathbb{Z} / 2 \mathbb{Z}$. The sextuple $\left(\mathcal{A}, \mathcal{A}_{+}, \mathbb{Z}_{2}, \alpha, \Theta, \tau\right)$ forms the field system in the chemical potential theory. By the evenness of $\phi$, its stabilizer

$$
G_{\phi}:=\left\{m \in \mathbb{Z}_{2}: \phi \circ \Theta^{m}=\phi\right\}
$$

coincides with $\mathbb{Z}_{2}$. Since its asymmetry subgroup

$$
N_{\phi}:=\left\{m \in \mathbb{Z}_{2}: \phi\left(a \Theta^{m}(b)\right)=\phi(a b) \text { for all } a, b \in \mathcal{A}\right\}
$$


is $\{0\}$, the $N_{\phi}$-fixed point subalgebra $\mathcal{A}^{N_{\phi}}$ coincides with $\mathcal{A}$. From [4, Sect. II.8], $\phi$ is an $\alpha$-KMS state of $\mathcal{A}$.

Step 4. We show that $\omega$ extends uniquely to a $\tau$-invariant $\alpha$-KMS state of $\mathcal{A}$. Since $\mathcal{A}$ is separable, $K_{\tau}(\mathcal{A})$ and $K_{\tau}\left(\mathcal{A}_{+}\right)$ are metrizable weak* compact convex sets in $\mathcal{A}^{*}$ and $\mathcal{A}_{+}^{*}, \mathcal{A}^{*}$ and $\mathcal{A}_{+}^{*}$ being the dual spaces of $\mathcal{A}$ and $\mathcal{A}_{+}$, respectively. By [15], $\mathscr{E} K_{\tau}(\mathcal{A})$ and $\mathscr{E} K_{\tau}\left(\mathcal{A}_{+}\right)$are $G_{\delta}$-sets. Let $f$ be a continuous linear mapping from $K_{\tau}\left(\mathcal{A}_{)}\right)$into $K_{\tau}\left(\mathcal{A}_{+}\right)$defined by $f(\rho):=\rho_{+}$for all $\rho \in K_{\tau}(\mathcal{A})$. By Step 3, $\mathcal{E} K_{\tau}\left(\mathcal{A}_{+}\right)$is contained in $Y:=f\left(K_{\tau}(\mathcal{A})\right)$. From [18], we can choose a measurable mapping $g$ from $Y$ into $K_{\tau}(\mathcal{A})$ such that $f \circ g(\psi)=\psi$ for all $\psi \in Y$. Let

$$
\bar{\omega}(a):=\int_{E K_{\tau}\left(\mathcal{A}_{+}\right)} g(\psi)(a) \mathrm{d} \mu(\psi), \quad a \in \mathcal{A} .
$$

Then $\bar{\omega} \in K_{\tau}(\mathcal{A})$. For each $a \in \mathcal{A}_{+}$, we obtain

$$
\begin{aligned}
\bar{\omega}_{+}(a) & =\int_{\mathcal{E} K_{\tau}\left(\mathcal{A}_{+}\right)} f \circ g(\psi)(a) \mathrm{d} \mu(\psi) \\
& =\int_{\mathcal{E}_{\tau}\left(\mathcal{A}_{+}\right)} \psi(a) \mathrm{d} \mu(\psi)=\omega(a),
\end{aligned}
$$

where the second equality is due to $f \circ g=\mathrm{id}$ on $Y$ and the last equality is due to (3.3). The uniqueness of $\bar{\omega}$ follows from its evenness.

The following corollary says that [17, Lemma 4.7] is valid in the fermion case as well.

Corollary 3.2. $\mathscr{E} K_{\tau}(\mathcal{A}) \subset \mathcal{E} \mathfrak{S}_{\tau}(\mathcal{A})$.

Proof. For each $\omega \in \mathcal{E} K_{\tau}(\mathcal{A})$, let $\left(\pi_{\omega_{+}}, \mathscr{H}_{\omega_{+}}\right)$be the cyclic representation of $\mathcal{A}_{+}$induced by $\omega_{+}$. Let $\omega_{+}=$ $\lambda \omega_{1}+(1-\lambda) \omega_{2}$ with $\omega_{1}, \omega_{2} \in K_{\tau}\left(\mathcal{A}_{+}\right)$and $0<\lambda<1$. For $i=1,2$, from Theorem 3.1, there exists a unique $\bar{\omega}_{i}$ in $K_{\tau}(\mathcal{A})$ such that $\bar{\omega}_{i+}=\omega_{i}$. By the evenness of $\tau$-invariant states, we have $\omega=\lambda \bar{\omega}_{1}+(1-\lambda) \bar{\omega}_{2}$. By the extremality of $\omega$, we obtain $\omega=\bar{\omega}_{1}=\bar{\omega}_{2}$, and hence $\omega_{+} \in \mathcal{E} K_{\tau}\left(\mathcal{A}_{+}\right)$. Let $u_{\omega_{+}}$be a unitary representation of $\mathbb{Z}^{v}$ implementing $\tau$ on $\mathscr{H}_{\omega_{+}}$. It then follows that

$$
\pi_{\omega_{+}}\left(\mathcal{A}_{+}\right)^{\prime \prime} \cap \pi_{\omega_{+}}\left(\mathcal{A}_{+}\right)^{\prime} \cap u_{\omega_{+}}\left(\mathbb{Z}^{\nu}\right)^{\prime}=\mathbb{C} \mathbf{1}_{\omega_{+}} .
$$

By (2.1) and $[6,4.3 .17]$, we obtain $\omega_{+} \in \mathscr{E S}_{\tau}\left(\mathcal{A}_{+}\right)$. Now, from the evenness of $\tau$-invariant states, it is easy to see that $\omega \in \mathcal{E} \mathfrak{S}_{\tau}(\mathcal{A})$.

Corollary 3.3. If $\omega \in K_{\tau}(\mathcal{A})$, then $\omega$ is extremal in $K_{\tau}(\mathcal{A})$ if and only if it is extremal in $\mathfrak{S}_{\tau}(\mathcal{A})$.

Proof. By Corollary 3.2, it suffices to show that $\mathscr{E S}_{\tau}(\mathcal{A}) \cap K_{\tau}(\mathcal{A}) \subset \mathcal{E} K_{\tau}(\mathcal{A})$. But this is immediately seen.

In the remainder of this section let us confine ourselves to the one-dimensional case $v=1$. For each $n \in \mathbb{N}$, let $\mathrm{I}_{n}:=\{-n,-n+1, \ldots, n\}$. Let $\mathcal{B}_{0}$ denote the set of all potentials $\Phi$ satisfying $(\mathrm{P} 1),(\mathrm{P} 2),(\mathrm{P} 3),(\mathrm{P} 6)$ and the following conditions:

$$
\sum_{\mathrm{K} \ni 0}\|\Phi(\mathrm{K})\|<\infty \quad \text { and } \quad \sup _{n \in \mathbb{N}}\left\|W_{\Phi}([1, n])\right\|<\infty
$$

Then $\mathcal{B}_{0}$ is a real Banach space with the usual linear operations and the norm

$$
\|\Phi\|_{0}:=\sum_{\mathrm{K} \ni 0}\|\Phi(\mathrm{K})\|+\sup _{n \in \mathbb{N}}\left\|W_{\Phi}([1, n])\right\|<\infty .
$$

Let $\Phi \in \mathcal{B}_{0}$. Since $\left\{W_{\Phi}([1, n])\right\}$ is uniformly bounded, it follows from $[11,14]$ that the closure of $\delta_{\Phi}$ is a generator of a strongly continuous automorphism group $\alpha^{\Phi}$ of $\mathcal{A}$. Moreover, we have

$$
\lim _{n \rightarrow \infty}\left\|\alpha_{t}^{\Phi}(a)-e^{i t U_{\Phi}\left(\mathrm{I}_{n}\right)} a e^{-i t U_{\Phi}\left(\mathrm{I}_{n}\right)}\right\|=0, \quad a \in \mathcal{A},
$$

where the convergence is uniform in $t$ on every compact subset of $\mathbb{R}$. By (3.4), it is easily verified that $\left(\mathcal{A}, \alpha^{\Phi}\right)$ is a dynamics satisfying (I)-(IV). We denote by $K\left(\mathcal{A}_{+}, \Phi\right)$ the set of all $\left.\alpha^{\Phi}\right|_{\mathcal{A}_{+}}$-KMS states of $\mathcal{A}_{+}$.

For simplicity we denote by $\tau$ the translation $\tau_{1}$. For each $k \in \mathbb{Z}$, let $[k]$ denote its representative in $\mathrm{I}_{n}$, namely $[k] \in \mathrm{I}_{n}$ and $k \equiv[k](\bmod 2 n+1)$, and let $\bar{\tau}$ be the periodic translation defined by $\bar{\tau}\left(a_{i}\right):=a_{[i+1]}$ for all $i \in \mathrm{I}_{n}$.

Lemma 3.4. If $\Phi \in \mathscr{B}_{0}$ and $\omega \in K\left(\mathcal{A}_{+}, \Phi\right)$, then $\omega$ is $\tau$-invariant.

Proof. Since $\mathcal{A}\left(\mathrm{I}_{n}\right)$ is a factor of type $\mathrm{I}$, there exists a unitary $u_{n} \in \mathcal{A}\left(\mathrm{I}_{n}\right)$ such that $\bar{\tau}(a)=u_{n} a u_{n}^{*}$ for all $a \in \mathcal{A}\left(\mathrm{I}_{n}\right)$. Since $\bar{\tau}$ commutes with $\Theta$, we have

$$
u_{n} \Theta(a) u_{n}^{*}=\bar{\tau}(\Theta(a))=\Theta(\bar{\tau}(a))=\Theta\left(u_{n}\right) \Theta(a) \Theta\left(u_{n}\right)^{*}, \quad a \in \mathcal{A}\left(\mathrm{I}_{n}\right) .
$$

Thus $\Theta\left(u_{n}\right)^{*} u_{n}$ is a scalar multiple of $\mathbf{1}$, and hence there exists a complex number $\lambda$ with modulus 1 such that 
$\Theta\left(u_{n}\right)=\lambda u_{n}$. Since $u_{n}=\Theta\left(\Theta\left(u_{n}\right)\right)=\lambda \Theta\left(u_{n}\right)=\lambda^{2} u_{n}$ due to $\Theta^{2}=\mathrm{id}$, we obtain $\lambda= \pm 1$. This implies that $u_{n}$ is either even or odd. Suppose that $u_{n}$ is odd. Let $w_{n+1}:=a_{n+1}+a_{n+1}^{*}$ and $v_{n}:=u_{n} w_{n+1}$. Then $w_{n+1}$ is a self-adjoint unitary in $\mathcal{A}(\{n+1\})_{-}$and $v_{n}$ is a unitary in $\mathcal{A}([-n, n+1])_{+}$. Since $a \in \mathcal{A}\left(\mathrm{I}_{n}\right)_{+}$commutes with $w_{n+1}$, we obtain

$$
v_{n} a v_{n}^{*}=u_{n} w_{n+1} a w_{n+1} u_{n}^{*}=u_{n} a u_{n}^{*}=\bar{\tau}(a), \quad a \in \mathcal{A}\left(\mathrm{I}_{n}\right)_{+} .
$$

Replacing $u_{n}$ by $v_{n}$, we may assume that $u_{n}$ is even. Since $\left\{\mathrm{I}_{n}\right\}$ tends to $\mathbb{Z}$ whenever $n \rightarrow \infty$, it follows that

$$
\tau(a)=\lim _{n \rightarrow \infty} u_{n} a u_{n}^{*}, \quad a \in \mathcal{A}
$$

in the norm topology. Here, notice that $\alpha_{t}^{\Phi} \tau=\tau \alpha_{t}^{\Phi}$ for all $t \in \mathbb{R}$. It is obvious that $\left\{u_{n}\right\}$ is contained in $\mathscr{D}\left(\delta_{\alpha^{\Phi}}\right)$. Moreover, in the same way as in the proof of [1, Proposition 1.1], we can show that $\sup _{n \in \mathbb{N}}\left\|\delta_{\alpha^{\Phi}}\left(u_{n}\right)\right\|<\infty$. Now, we can apply $\left[9\right.$, Corollary II.3] (or $[6,5.3 .33 \mathrm{~A}]$ ) to conclude that if $\omega$ is an $\left.\alpha^{\Phi}\right|_{\mathcal{A}_{+}}$-KMS state of $\mathcal{A}_{+}$, then $\omega \circ \tau=\omega$.

Corollary 3.5. If $\Phi \in \mathscr{B}_{0}$, then $\mathcal{A}_{+}$has a unique $\left.\alpha^{\Phi}\right|_{\mathcal{A}_{+}}-K M S$ state.

Proof. Each $\omega \in K\left(\mathcal{A}_{+}, \Phi\right)$ extends uniquely to an $\alpha^{\Phi}$-KMS state $\bar{\omega}$ of $\mathcal{A}$ due to Lemma 3.4 and Theorem 3.1. Since $\Phi \in \mathscr{B}_{0}$, it follows from $\left[6,6.2\right.$.47] (see also $[2,12]$ ) that $\mathcal{A}$ has a unique $\alpha^{\Phi}$-KMS state $\psi$. Hence we obtain $\omega=\psi_{+}$, and thus $K\left(\mathscr{A}_{+}, \Phi\right)$ is a one-point set $\left\{\psi_{+}\right\}$.

\section{Macroscopic Uniformity}

Let $\alpha$ be a one-parameter dynamics on the fermion algebra $\mathcal{A}$ over $\mathbb{Z}^{v}$ as mentioned in Introduction. In this section, let $\delta$ be the restriction of $\delta_{\alpha}$ to $\mathcal{A}_{0}$, and let $\Phi$ denote the unique $\tau$-invariant standard potential determined by $\delta$ [5, Corollary 8.5]. To introduce the Gibbs condition, we need the notion of perturbations of states of $\mathcal{A}$. Let $\psi, \omega \in \mathfrak{S}(\mathcal{A})$. For each $\mathrm{I} \in \mathscr{F}$, the relative entropy of $\psi_{\mathrm{I}}$ with respect to $\omega_{\mathrm{I}}$ is given by

$$
S\left(\psi_{\mathrm{I}}, \omega_{\mathrm{I}}\right):=\operatorname{Tr}_{\mathrm{I}}\left(\frac{\mathrm{d} \psi_{\mathrm{I}}}{\mathrm{d} \operatorname{Tr}_{\mathrm{I}}}\left(\log \frac{\mathrm{d} \psi_{\mathrm{I}}}{\mathrm{d} \operatorname{Tr}_{\mathrm{I}}}-\log \frac{\mathrm{d} \omega_{\mathrm{I}}}{\mathrm{d} \operatorname{Tr}_{\mathrm{I}}}\right)\right) .
$$

Then the relative entropy $S(\psi, \omega)$ is defined by

$$
S(\psi, \omega):=\sup _{\mathrm{I} \subset \mathbb{Z}^{v}} S\left(\psi_{\mathrm{I}}, \omega_{\mathrm{I}}\right)=\lim _{\mathrm{I} \nearrow \mathbb{Z}^{v}} S\left(\psi_{\mathrm{I}}, \omega_{\mathrm{I}}\right) .
$$

For each $\omega \in \mathfrak{S}(\mathcal{A})$ and $h=h^{*} \in \mathcal{A}$, since $\psi \mapsto S(\psi, \omega)+\psi(h)$ is weakly* lower semicontinuous and strictly convex on $\mathfrak{S}(\mathcal{A})$, the perturbed state $\left[\omega^{h}\right]$ by $h$ is defined as a unique minimizer of this functional [8]. Recall $[3,8]$ that

$$
\left|S(\psi, \omega)-S\left(\psi,\left[\omega^{h}\right]\right)\right| \leq 2\|h\|
$$

for every $\psi, \omega \in \mathfrak{S}(\mathcal{A})$ and $h=h^{*} \in \mathcal{A}$.

Let $\omega \in \mathfrak{S}(\mathcal{A})$ and $\left(\pi_{\omega}, \mathscr{H}_{\omega}, \Omega_{\omega}\right)$ be the cyclic representation of $\mathcal{A}$ induced by $\omega$. We denote by $\left[\omega^{-W_{\Phi}(\mathrm{I})}\right]^{\sim}$ the normal extension of $\left[\omega^{-W_{\Phi}(\mathrm{I})}\right]$ to $\pi_{\omega}(\mathcal{A})^{\prime \prime}$. A state $\omega$ of $\mathcal{A}$ is said to satisfy the $\delta$-Gibbs condition, or alternatively the $\Phi$-Gibbs condition if $\Omega_{\omega}$ is separating for $\pi_{\omega}(\mathcal{A})^{\prime \prime}$ and if, for each $\mathrm{I} \in \mathscr{F}$,

$$
\sigma_{t}^{\left[\omega^{-W_{\Phi}(\mathrm{I})}\right]^{\tau}}\left(\pi_{\omega}(a)\right)=\pi_{\omega}\left(e^{-i t U_{\Phi}(\mathrm{I})} a e^{i t U_{\Phi}(\mathrm{I})}\right), \quad a \in \mathcal{A}(\mathrm{I}) .
$$

Here, $\sigma_{t}^{\left[\omega^{-W_{\Phi}(\mathrm{I})}\right]^{\sim}}$ denotes the modular automorphism group for $\left[\omega^{-W_{\Phi}(\mathrm{I})}\right]^{\sim}$ (this definition is due to [5]).

If $\omega$ is a state of $\mathcal{A}$, then the following conditions are equivalent [5, Sect. 7.4]:

(i) $\omega$ is an $\alpha$-KMS state.

(ii) $\omega$ satisfies the $\delta$-Gibbs condition.

For each $\mathrm{I} \in \mathscr{F}$, the local Gibbs state $\phi_{\mathrm{I}}^{\mathrm{G}}$ of $\mathcal{A}(\mathrm{I})$ with respect to $\delta$ is defined by

$$
\phi_{\mathrm{I}}^{\mathrm{G}}(a):=\frac{\operatorname{Tr}_{\mathrm{I}}\left(e^{-U_{\Phi}(\mathrm{I})} a\right)}{\operatorname{Tr}_{\mathrm{I}}\left(e^{-U_{\Phi}(\mathrm{I})}\right)}, \quad a \in \mathcal{A}(\mathrm{I}) .
$$

Let $\mathrm{I} \in \mathscr{F}, \mathrm{I} \neq \emptyset$ and $\omega$ be a $\tau$-invariant state of $\mathcal{A}$ satisfying the $\delta$-Gibbs condition. Since $\omega$ is even, it follows from [5, Proposition 7.7] that

$$
\left[\omega^{-W_{\Phi}(\mathrm{I})}\right](a b)=\phi_{\mathrm{I}}^{\mathrm{G}}(a)\left[\omega^{-W_{\Phi}(\mathrm{I})}\right](b), \quad a \in \mathcal{A}(\mathrm{I}), b \in \mathcal{A}\left(\mathrm{I}^{c}\right) .
$$

To establish the macroscopic uniformity of an extremal $\tau$-invariant state of $\mathcal{A}$ satisfying the $\delta$-Gibbs condition, we need some auxiliary results.

Lemma 4.1. If $\omega$ is a $\tau$-invariant state of $\mathcal{A}$ satisfying the $\delta$-Gibbs condition, then

$$
\log \frac{\mathrm{d} \phi_{\mathrm{I}}^{\mathrm{G}}}{\mathrm{d} \operatorname{Tr}_{\mathrm{I}}}-\log \frac{\mathrm{d} \omega_{\mathrm{I}}}{\mathrm{d} \operatorname{Tr}_{\mathrm{I}}} \leq 2\left\|W_{\Phi}(\mathrm{I})\right\| \mathbf{1}
$$

for every $\mathrm{I} \in \mathscr{F}$. 
Proof. For every $\psi \in \mathfrak{S}(\mathcal{A})$, let $\tilde{\psi}_{\mathrm{I}}$ be the state of $\pi_{\omega}(\mathcal{A}(\mathrm{I}))$ defined by $\tilde{\psi}_{\mathrm{I}}\left(\pi_{\omega}(a)\right):=\psi_{\mathrm{I}}(a)$ for all $a \in \mathcal{A}(\mathrm{I})$. In particular, let $\tilde{\phi}_{\mathrm{I}}^{\mathrm{G}}$ be that for $\phi_{\mathrm{I}}^{\mathrm{G}}$. From the $\delta$-Gibbs condition, we obtain

$$
\sigma_{t}^{\left[\omega^{-W_{\Phi}(\mathrm{I})}\right]^{\sim}}\left(\pi_{\omega}(\mathcal{A}(\mathrm{I}))\right)=\pi_{\omega}(\mathcal{A}(\mathrm{I})), \quad t \in \mathbb{R} .
$$

Hence [16] implies that there exists a conditional expectation $\varepsilon_{\mathrm{I}}$ from $\pi_{\omega}(\mathcal{A})^{\prime \prime}$ onto $\pi_{\omega}(\mathcal{A}(\mathrm{I}))$ with respect to $\left[\omega^{-W_{\Phi}(\mathrm{I})}\right]^{\sim}$. Let $\tilde{\omega}$ be the normal extension of $\omega$ to $\pi_{\omega}(\mathcal{A})^{\prime \prime}$ and $\tilde{\omega}_{\mathrm{I}}:=\left.\tilde{\omega}\right|_{\pi_{\omega}(\mathcal{A}(\mathrm{I}))}$. Note that $\left[\omega^{-W_{\Phi}(\mathrm{I})}\right]^{\sim}=\left[\tilde{\omega}^{-\pi_{\omega}\left(W_{\Phi}(\mathrm{I})\right)}\right]$. It follows from (4.3) that $\left[\omega^{-W_{\Phi}(\mathrm{I})}\right]^{\sim}=\left[\omega^{-W_{\Phi}(\mathrm{I})}\right]^{\sim} \circ \varepsilon_{\mathrm{I}}=\tilde{\phi}_{\mathrm{I}}^{\mathrm{G}} \circ \varepsilon_{\mathrm{I}}$. Then we have

$$
\begin{aligned}
S\left(\psi_{\mathrm{I}}, \omega_{\mathrm{I}}\right) & =S\left(\tilde{\psi}_{\mathrm{I}}, \tilde{\omega}_{\mathrm{I}}\right) \\
& \leq S\left(\tilde{\psi}_{\mathrm{I}} \circ \varepsilon_{\mathrm{I}}, \tilde{\omega}\right) \\
& \leq S\left(\tilde{\psi}_{\mathrm{I}} \circ \varepsilon_{\mathrm{I}},\left[\omega^{-W_{\Phi}(\mathrm{I})}\right]^{\sim}\right)+2\left\|W_{\Phi}(\mathrm{I})\right\| \\
& =S\left(\tilde{\psi}_{\mathrm{I}} \circ \varepsilon_{\mathrm{I}}, \tilde{\phi}_{\mathrm{I}}^{\mathrm{G}} \circ \varepsilon_{\mathrm{I}}\right)+2\left\|W_{\Phi}(\mathrm{I})\right\| \\
& =S\left(\psi_{\mathrm{I}}, \phi_{\mathrm{I}}^{\mathrm{G}}\right)+2\left\|W_{\Phi}(\mathrm{I})\right\|,
\end{aligned}
$$

where the first inequality is due to the monotonicity of relative entropy and the second inequality is due to (4.1). Hence

$$
\psi\left(\log \frac{\mathrm{d} \phi_{\mathrm{I}}^{\mathrm{G}}}{\mathrm{d} \operatorname{Tr}_{\mathrm{I}}}-\log \frac{\mathrm{d} \omega_{\mathrm{I}}}{\mathrm{d} \operatorname{Tr}_{\mathrm{I}}}\right) \leq 2\left\|W_{\Phi}(\mathrm{I})\right\|
$$

for all $\psi \in \mathfrak{S}(\mathcal{A})$. This implies the conclusion.

If $\Psi$ is a $\tau$-invariant standard potential having relatively short range, i.e.,

$$
\sum_{\mathrm{K} \ni 0} \frac{\|\Psi(\mathrm{K})\|}{|\mathrm{K}|}<\infty
$$

then we can define the mean energy operator $A_{\Psi}$ of $\Psi$ by

$$
A_{\Psi}:=\sum_{\mathrm{K} \ni 0} \frac{\Psi(\mathrm{K})}{|\mathrm{K}|} .
$$

Since $\Psi$ is $\Theta$-invariant, so is $A_{\Psi}$.

Lemma 4.2. If $\psi \in \mathfrak{S}_{\tau}(\mathcal{A})$ and $\Psi \in \mathcal{P}_{\tau}$ has relatively short range, then $e_{\Psi}(\psi)=\psi\left(A_{\Psi}\right)$.

Proof. Let $\mathrm{I} \in \mathscr{F}$. Since

$$
\begin{aligned}
\left|\frac{\psi\left(U_{\Psi}(\mathrm{I})\right)}{|\mathrm{I}|}-\psi\left(A_{\Psi}\right)\right| & \leq \frac{1}{|\mathrm{I}|}\left\|\sum_{\mathrm{K} \subset \mathrm{I}} \Psi(\mathrm{K})-\sum_{k \in \mathrm{I}} \tau_{k}\left(A_{\Psi}\right)\right\| \\
& \leq \frac{1}{|\mathrm{I}|} \sum_{k \in \mathrm{I}} \sum_{\substack{\mathrm{K} \cap \mathrm{I}^{c} \neq \emptyset \\
\mathrm{K} \ni k}} \frac{\|\Psi(\mathrm{K})\|}{|\mathrm{K}|},
\end{aligned}
$$

we obtain $e_{\Psi}(\psi)=\psi\left(A_{\Psi}\right)$ in the same way as [6, 6.2.39].

Lemma 4.3. If $\psi \in \mathcal{E S}_{\tau}(\mathcal{A})$ and $\Psi \in \mathcal{P}_{\tau}$ has relatively short range, then

$$
\text { v.H. } \lim _{\mathrm{I} \rightarrow \mathbb{Z}^{v}} \frac{1}{|\mathrm{I}|} \pi_{\psi}\left(U_{\Psi}(\mathrm{I})\right)=e_{\Psi}(\psi) \mathbf{1}
$$

in the strong operator topology.

Proof. From the proof of Lemma 4.2, we have

$$
\text { v.H. } \lim _{\mathrm{I} \rightarrow \mathbb{Z}^{v}} \frac{1}{|\mathrm{I}|} U_{\Psi}(\mathrm{I})=\text { v.H. } \lim _{\mathrm{I} \rightarrow \mathbb{Z}^{v}} \frac{1}{|\mathrm{I}|} \sum_{k \in \mathrm{I}} \tau_{k}\left(A_{\Psi}\right)
$$

in the norm topology. Using the mean ergodic theorem (see e.g. [7]) and [5, Lemma A.5], we have

$$
\text { v.H. } \lim _{\mathrm{I} \rightarrow \mathbb{Z}^{v}} \frac{1}{|\mathrm{I}|} \sum_{k \in \mathrm{I}} \pi_{\psi}\left(\tau_{k}\left(A_{\Psi}\right)\right)=\psi\left(A_{\Psi}\right) \mathbf{1}
$$

in the strong operator topology. By (4.4), (4.5) and Lemma 4.2, we obtain the conclusion.

We shall remove the assumption of relatively short range as follows.

Lemma 4.4. If $\omega \in \mathfrak{E S}_{\tau}(\mathcal{A})$, then

$$
\text { v.H. } \lim _{\mathrm{I} \rightarrow \mathbb{Z}^{v}} \frac{1}{|\mathrm{I}|} \pi_{\omega}\left(U_{\Phi}(\mathrm{I})\right)=e_{\Phi}(\omega) \mathbf{1}
$$


in the strong operator topology.

Proof. Since $\mathcal{P}_{\tau}^{\mathrm{f}}$ is dense in $\mathcal{P}_{\tau}$, there exists a sequence $\left\{\Phi_{n}\right\}$ in $\mathcal{P}_{\tau}^{\mathrm{f}}$ such that $\left\{\Phi_{n}\right\}$ is convergent to $\Phi$ in the norm topology. Given any $\varepsilon>0$, there exists a positive integer $N$ such that $\left\|\Phi_{n}-\Phi\right\| \leq \varepsilon$ whenever $n \geq N$. Let $\left\{\mathrm{I}_{\alpha}\right\}$ be a van Hove net tending to $\mathbb{Z}^{v}$. By (2.3), we have

$$
\begin{aligned}
\left\|\frac{1}{\left|\mathrm{I}_{\alpha}\right|} \pi_{\omega}\left(U_{\Phi_{n}}\left(\mathrm{I}_{\alpha}\right)\right)-\frac{1}{\left|\mathrm{I}_{\alpha}\right|} \pi_{\omega}\left(U_{\Phi}\left(\mathrm{I}_{\alpha}\right)\right)\right\| & =\frac{1}{\left|\mathrm{I}_{\alpha}\right|}\left\|U_{\Phi_{n}-\Phi}\left(\mathrm{I}_{\alpha}\right)\right\| \\
& \leq\left\|\Phi_{n}-\Phi\right\| \leq \varepsilon
\end{aligned}
$$

whenever $n \geq N$. Note that

$$
\left|e_{\Phi_{n}}(\omega)-e_{\Phi}(\omega)\right|=\left|e_{\Phi_{n}-\Phi}(\omega)\right| \leq\left\|\Phi_{n}-\Phi\right\| \leq \varepsilon
$$

for all $n \geq N$. By Lemma 4.3, we can choose an index $\alpha(N)$ of the net $\left\{\mathrm{I}_{\alpha}\right\}$ such that

$$
\left\|\frac{1}{\left|\mathrm{I}_{\alpha}\right|} \pi_{\omega}\left(U_{\Phi_{N}}\left(\mathrm{I}_{\alpha}\right)\right)-e_{\Phi_{N}}(\omega) \mathbf{1}\right\| \leq \varepsilon
$$

whenever $\alpha \geq \alpha(N)$. Hence, by (4.6), (4.7) and (4.8), for $\alpha \geq \alpha(N)$ we have

$$
\begin{aligned}
& \left\|\frac{1}{\left|\mathrm{I}_{\alpha}\right|} \pi_{\omega}\left(U_{\Phi}\left(\mathrm{I}_{\alpha}\right)\right)-e_{\Phi}(\omega) \mathbf{1}\right\| \\
& \quad \leq\left\|\frac{1}{\left|\mathrm{I}_{\alpha}\right|} \pi_{\omega}\left(U_{\Phi}\left(\mathrm{I}_{\alpha}\right)\right)-\frac{1}{\left|\mathrm{I}_{\alpha}\right|} \pi_{\omega}\left(U_{\Phi_{N}}\left(\mathrm{I}_{\alpha}\right)\right)\right\| \\
& \quad+\left\|\frac{1}{\left|\mathrm{I}_{\alpha}\right|} \pi_{\omega}\left(U_{\Phi_{N}}\left(\mathrm{I}_{\alpha}\right)\right)-e_{\Phi_{N}}(\omega) \mathbf{1}\right\|+\left|e_{\Phi_{N}}(\omega)-e_{\Phi}(\omega)\right| \leq 3 \varepsilon .
\end{aligned}
$$

This estimation implies the conclusion.

Proposition 4.5. If $\omega$ is an extremal $\tau$-invariant state of $\mathcal{A}$ satisfying the $\delta$-Gibbs condition, then

$$
\text { v.H. } \lim _{\mathrm{I} \rightarrow \mathbb{Z}^{v}|\mathrm{I}|} \frac{1}{\omega} \pi_{\omega}\left(-\log \frac{\mathrm{d} \omega_{\mathrm{I}}}{\mathrm{d} \mathrm{Tr}_{\mathrm{I}}}\right)=\text { v.H. } \lim _{\mathrm{I} \rightarrow \mathbb{Z}^{v}} \frac{1}{|\mathrm{I}|} \pi_{\omega}\left(-\log \frac{\mathrm{d} \phi_{\mathrm{I}}^{\mathrm{G}}}{\mathrm{d} \operatorname{Tr}_{\mathrm{I}}}\right)=s(\omega) \mathbf{1}
$$

in the strong operator topology.

Proof. By (4.2), we obtain

$$
\pi_{\omega}\left(-\log \frac{\mathrm{d} \phi_{\mathrm{I}}^{\mathrm{G}}}{\mathrm{d} \operatorname{Tr}_{\mathrm{I}}}\right)=\pi_{\omega}\left(U_{\Phi}(\mathrm{I})\right)+\log \operatorname{Tr}_{\mathrm{I}}\left(e^{-U_{\Phi}(\mathrm{I})}\right) \mathbf{1} .
$$

By dividing the above equality by $|\mathrm{I}|$ and then taking the van Hove limit $\mathrm{I} \rightarrow \mathbb{Z}^{v}$, we obtain the following strong convergence

$$
\text { v.H. } \lim _{\mathrm{I} \rightarrow \mathbb{Z}^{v}} \frac{1}{|\mathrm{I}|} \pi_{\omega}\left(-\log \frac{\mathrm{d} \phi_{\mathrm{I}}^{\mathrm{G}}}{\mathrm{d} \operatorname{Tr}_{\mathrm{I}}}\right)=e_{\Phi}(\omega) \mathbf{1}+P(\Phi) \mathbf{1}=s(\omega) \mathbf{1},
$$

where the first equality is due to Lemma 4.4 and the second equality follows from the variational principle of $\omega$ [5, Proposition 12.1].

Now let

$$
a_{\mathrm{I}}:=-\frac{1}{|\mathrm{I}|} \log \frac{\mathrm{d} \omega_{\mathrm{I}}}{\mathrm{d} \operatorname{Tr}_{\mathrm{I}}}, \quad b_{\mathrm{I}}:=-\frac{1}{|\mathrm{I}|} \log \frac{\mathrm{d} \phi_{\mathrm{I}}^{\mathrm{G}}}{\mathrm{d} \operatorname{Tr}_{\mathrm{I}}}+\frac{2\left\|W_{\Phi}(\mathrm{I})\right\|}{|\mathrm{I}|} \mathbf{1} .
$$

It follows from [5, Lemma 9.1] that

$$
\text { v.H. } \lim _{\mathrm{I} \rightarrow \mathbb{Z}^{v}} \frac{\left\|W_{\Phi}(\mathrm{I})\right\|}{|\mathrm{I}|}=0 .
$$

From this fact and (4.9), $\pi_{\omega}\left(b_{\mathrm{I}}\right) \rightarrow s(\omega) \mathbf{1}$ in the strong operator topology. By Lemma 4.1, we have $0 \leq a_{\mathrm{I}} \leq b_{\mathrm{I}}$. Since $\left\{b_{\mathrm{I}}\right\}$ is uniformly bounded, so is $\left\{b_{\mathrm{I}}-a_{\mathrm{I}}\right\}$. Let $\tilde{\omega}$ be the normal extension of $\omega$ to $\pi_{\omega}(\mathcal{A})^{\prime \prime}$. Since $\omega$ satisfies the $\delta$-Gibbs condition, $\Omega_{\omega}$ is separating for $\pi_{\omega}(\mathcal{A})^{\prime \prime}$. Hence $\tilde{\omega}$ is automatically faithful. Then we have

$$
\text { v.H. } \lim _{\mathrm{I} \rightarrow \mathbb{Z}^{v}} \tilde{\omega}\left(\pi_{\omega}\left(b_{\mathrm{I}}-a_{\mathrm{I}}\right)\right)=\text { v.H. } \lim _{\mathrm{I} \rightarrow \mathbb{Z}^{v}} \omega\left(b_{\mathrm{I}}-a_{\mathrm{I}}\right)=0 .
$$

By the faithfulness of $\tilde{\omega}$, we obtain

$$
\text { v.H. } \lim _{\mathrm{I} \rightarrow \mathbb{Z}^{v}} \pi_{\omega}\left(a_{\mathrm{I}}\right)=\text { v.H. } \lim _{\mathrm{I} \rightarrow \mathbb{Z}^{v}} \pi_{\omega}\left(b_{\mathrm{I}}\right)=s(\omega) \mathbf{1}
$$

in the strong operator topology. 
To study the macroscopic uniformity of extremal equilibrium states in a quantum spin system, Hiai and Petz introduced the quantity defined as follows: Let $0<\varepsilon<1$. For each $\mathrm{I} \in \mathscr{F}$ and each state $\omega$ of $\mathcal{A}(\mathrm{I})$, define

$$
\beta_{\varepsilon}(\omega):=\min \left\{\operatorname{Tr}_{\mathrm{I}}(q): q \in \mathcal{A}(\mathrm{I}) \text { is a projection with } \omega(q) \geq 1-\varepsilon\right\}
$$

(see [10]).

Theorem 4.6. If $\omega$ is an extremal $\tau$-invariant state of $\mathcal{A}$ satisfying the $\delta$-Gibbs condition, then

$$
\text { v.H. } \lim _{\mathrm{I} \rightarrow \mathbb{Z}^{v}} \frac{1}{|\mathrm{I}|} \log \beta_{\varepsilon}\left(\omega_{\mathrm{I}}\right)=s(\omega)
$$

for every $0<\varepsilon<1$.

Proof. This follows from Proposition 4.5 and the same argument as in the proof of [10, Theorem 3.1].

Remark 4.7. Assume that $\left\{W_{\Phi}\left(\mathrm{I}_{\alpha}\right)\right\}$ is uniformly bounded for some van Hove net $\left\{\mathrm{I}_{\alpha}\right\}$. Let $\omega$ be an even state of $\mathcal{A}$ satisfying the $\delta$-Gibbs condition. Since such $\omega$ is unique, it is extremal in $\mathfrak{S}_{\tau}(\mathcal{A})$ by Corollary 3.2. Then the following equalities are valid:

$$
\text { v.H. } \lim _{\mathrm{I} \rightarrow \mathbb{Z}^{v}} \frac{1}{|\mathrm{I}|} \log \beta_{\varepsilon}\left(\omega_{\mathrm{I}}\right)=s(\omega)=\text { v.H. } \lim _{\mathrm{I} \rightarrow \mathbb{Z}^{v}} \frac{1}{|\mathrm{I}|} \log \beta_{\varepsilon}\left(\phi_{\mathrm{I}}^{\mathrm{G}}\right)
$$

for every $0<\varepsilon<1$. The proof is the same as that of [10, Theorem 3.3].

\section{Appendix A: General Potentials}

A mapping $\Phi$ from $\mathscr{F}$ into $\mathcal{A}$ is called a general potential if it satisfies (P1), (P2), (P3) and (P5). For each general potential $\Phi$, we can define $H_{\Phi}(\mathrm{I}), U_{\Phi}(\mathrm{I}), W_{\Phi}(\mathrm{I})$ and $\delta_{\Phi}$ in the same way as the standard case. Two general potentials $\Phi$ and $\Psi$ are said to be physically equivalent if $\delta_{\Phi}=\delta_{\Psi}$. If $\Phi$ is a $\tau$-invariant general potential, then $\delta_{\Phi} \in \Delta_{\tau}\left(\mathcal{A}_{0}\right)$, and hence there is a unique $\Phi_{s} \in \mathcal{P}_{\tau}$ being physically equivalent to $\Phi$ [5, Corollary 8.5]. For each $\tau$-invariant general potential $\Phi$, let $\Phi_{s}$ denote the standard potential associated with $\Phi$. Let $\mathscr{B}_{1}$ denote the set of all potentials satisfying (P1), (P2), (P3) and of short range in the sense that

$$
\|\Phi\|_{1}:=\sum_{\mathrm{K} \ni 0}\|\Phi(\mathrm{K})\|<\infty .
$$

Then $\mathcal{B}_{1}$ becomes a real Banach space with the usual linear operations and the norm $\|\cdot\|_{1}$. Each $\Phi \in \mathscr{B}_{1}$ is a $\tau$ invariant general potential since (A.1) implies (P5) automatically.

Proposition A.1. The equation $S \Phi:=\Phi_{s}\left(\Phi \in \mathscr{B}_{1}\right)$ defines a bounded linear mapping $S$ from $\mathscr{B}_{1}$ into $\mathcal{P}_{\tau}$.

Proof. Since the linearity of $S$ is obvious, we show that $S$ is bounded. For this, suppose that a sequence $\left\{\Phi_{n}\right\}$ in $\mathscr{B}_{1}$ converges to $\Phi \in \mathscr{B}_{1}$ while $\left\{S \Phi_{n}\right\}$ converges to $\Psi \in \mathcal{P}_{\tau}$; we have to show that $S \Phi=\Psi$. Let $\Psi_{n}:=S \Phi_{n}$ for all $n \in \mathbb{N}$, and let $\mathrm{I} \in \mathscr{F}$. Then we have

$$
\left\|H_{\Phi_{n}}(\mathrm{I})-H_{\Phi}(\mathrm{I})\right\| \leq\left\|\Phi_{n}-\Phi\right\|_{1} \cdot|\mathrm{I}| \rightarrow 0
$$

as $n \rightarrow \infty$. By (2.3), we have

$$
\left\|H_{\Psi_{n}}(\mathrm{I})-H_{\Psi}(\mathrm{I})\right\| \leq\left\|\Psi_{n}-\Psi\right\| \cdot|\mathrm{I}| \rightarrow 0
$$

as $n \rightarrow \infty$. Hence $\delta_{\Psi}=\delta_{\Phi}$; so $S \Phi=\Psi$. From the closed graph theorem, $S$ is bounded.

Acknowledgments. The author is deeply grateful to Professor Fumio Hiai for his encouragement and many helpful suggestions, and also to a referee for useful comments.

\section{REFERENCES}

[1] Akiho, N., Hiai, F., and Petz, D., "Equilibrium states and their entropy densities in gauge-invariant $\mathrm{C}^{*}$-systems," Rev. Math. Phys., 17: 365-389 (2005).

[2] Araki, H., "On uniqueness of KMS states of one-dimensional quantum lattice systems," Commun. Math. Phys., 44: 1-7 (1975).

[3] Araki, H., "Relative entropy for states of von Neumann algebras II," Publ. Res. Inst. Math. Sci., 13: 173-192 (1977).

[4] Araki, H., Haag, R., Kastler, D., and Takesaki, M., "Extension of KMS states and chemical potential," Commun. Math. Phys., 53: 97-134 (1977).

[5] Araki, H., and Moriya, H., "Equilibrium statistical mechanics of Fermion lattice systems," Rev. Math. Phys., 15: 93-198 (2003).

[6] Bratteli, O., and Robinson, D. W., Operator Algebras and Quantum Statistical Mechanics 1, 2, 2nd edition, Springer-Verlag, 2002. 
[7] Conze, J. P., and Dang-Ngoc, N., "Ergodic theorems for noncommutative dynamical systems," Invent. Math., 46: 1-15 (1978).

[8] Donald, M. J., "Relative Hamiltonians which are not bounded from above," J. Funct. Anal., 91: 143-173 (1990).

[9] Fannes, M., Vanheuverzwijn, P., and Verbeure, A., "Quantum energy-entropy inequalities: A new method for proving the absence of symmetry breaking," J. Math. Phys., 25: 76-78 (1984).

[10] Hiai, F., and Petz, D., "Entropy densities for Gibbs states of quantum spin systems," Rev. Math. Phys., 5: 693-712 (1993).

[11] Kishimoto, A., "Dissipations and derivations," Commun. Math. Phys., 47: 25-32 (1976).

[12] Kishimoto, A., "On uniqueness of KMS states of one-dimensional quantum lattice systems," Commun. Math. Phys., 47: 167170 (1976).

[13] Narnhofer, H., and Thirring, W., "Spontaneously broken symmetries," Ann. Inst. Henri Poincaré, Phys. Théor., 70: 1-21 (1999).

[14] Powers, R. T., and Sakai, S., "Unbounded derivations in operator algebras," J. Funct. Anal., 19: 81-95 (1975).

[15] Phelps, R. R., Lectures on Choquet's Theorem, 2nd edition, Lecture Notes in Mathematics, 1757, Springer-Verlag, 2001.

[16] Takesaki, M., "Conditional expectations in von Neumann algebras," J. Funct. Anal., 9: 306-321 (1972).

[17] Takesaki, M., and Winnink, M., "Local normality in quantum statistical mechanics," Commun. Math. Phys., 30: 129-152 (1973).

[18] Takesaki, M., Theory of Operator Algebras I, Springer-Verlag, 1979. 